\title{
Clinical Possibilities of the Use of Stem Cells in Parodoncium Regeneration
}

\author{
Vukovic $\mathbf{M}^{*}$ \\ Faculty of Dentistry, Serbia \\ *Corresponding author: Mladen Vukovic, DMD, PhD candidate at the Faculty of Dentistry, Dr. Subotica 6, Street 11000 Belgrade, Serbia
}

Submission: 皆 January 09, 2019; Published: 監 January 30, 2019

\begin{abstract}
Stem cells are undifferentiated cells with varying degrees of potential and plasticity, capable of self-regeneration and multi-purpose differentiation. To date, stem cells have been isolated from three main sources: embryonic stem cells, somatic stem cells (adult stem cells, mesenchymal stem cells) and, more recently, through genetic manipulation of induced pluripotent stem cell (iPSc). While the use of embryonic stem cells is limited to ethical issues, somatic stem cells constitute a more favorable cellular source used in tissue engineering. Postnatal stem cells are isolated from several tissues including the brain, skin, hair follicles, skeletal muscles, bone marrow and dental tissue: Dental pulp stem cells (DPSC) from pulp of permanent teeth; stem cells from exfoliated deciduous teeth (SHED); periodontal ligament stem cells (PDLSCs); apical papilla stem cells (SCAP) and progenitor cells of dental follicles (DFPC). The use of stem cells in the treatment of various diseases of the oral cavity and diseased periodontium is gaining in importance. New treatments that involve tissue engineering, i.e. the use of stem cells for the regeneration of damaged dental tissues give new hope in solving this complex medical problem.

Abbreviations: iPSc: Induced Pluripotent Stem Cells; DPSC: Dental Pulp Stem Cells; SHED: Stem Cells from Exfoliated Teeth; PDLSC: Stem Cells of the Periodontal Ligament; SCAP: Apical Papilla Stem Cells; DFPC: Progenitor Cells of Dental Follicles; MSCs: Mesenchymal Stem Cells; HLA: Human Leukocytic Antigens; HA/TCP: Hydroxyapatite/Tricalcium Phosphate; MACS: Sorting of Magnetically Activated Cells; PDL: Periodontal Ligament; PLLA: Porous Poly-L-lactic Acid; NOD/SCID: Non-Obese Diabetic/Severe Combined Immunodeficiency; iPSCs-MSC: Mesenchymal Stem Cells Derived from iPSCs; EMDs: Spot Matrix Derivatives
\end{abstract}

\section{Introduction}

Parodontopathy is a chronic inflammatory disease of periodontium which can result in irreversible damage to the tissue, resulting in loss of contact between the tooth and its supporting device. Periodontal disease is the response of the immune system to the accumulation of plaque and oral microorganisms, which leads to a progressive loss of gingival tissue, periodontal ligament and alveolar bone. If it is not treated it can lead to premature loss of teeth. Parodontopathy is also associated with many systemic disorders such as diabetes, rheumatoid arthritis and cardiovascular complications, and its development is associated with a negative outcome of pregnancy. Due to all of this, periodontal disease significantly affects the patient's quality of life and has a significant financial impact on both the patient and the public health system. The ultimate goal of periodontal therapy is to achieve periodontal regeneration with the reconstruction of all parodontium components including periodontal ligament, connective gingival tissue, cement and alveolar bone. The treatments currently in use in the world and in our country can't completely reconstruct all of this tissues and relationships damaged by periodontal disease [1].

\section{Current approaches in the treatment of periodontal disease}

The complex nature of teeth and its structure make it difficult to develop effective and sustainable treatments for the treatment of periodontal disease. Nonetheless, many techniques and procedures have been developed to enable regeneration of diseased periodontium. In addition to conservative treatments, numerous approaches and techniques have been developed to stimulate the creation of a new bone at the defect site to compensate for bone loss resulting from periodontal disease. This includes the use of alloplastic materials, autografts, allografts, xenografts, etc. However, histological analysis of these treatments showed a minimum osteoinductive capacity. Recent tissue regeneration has become a widely accepted clinical procedure. This implies the use of a membrane barrier in order to help in the engagement of special cellular components of periodontium in regenerative processes. However, clinical improvements achieved by guided tissue regeneration are small and highly variable. Some treatments involved in the reconstruction of periodontal defects rely heavily on autologous tissue grafts and / or artificial implants. However, the success of these approaches was limited due to insufficient biocompatibility, bone resorption, limited amounts of grafts, etc. As a consequence, current research trends are focused on the development of periodontal regeneration techniques on the cell base [1].

\section{Tissue engineering}

Tissue engineering is a multidisciplinary field that combines biology, engineering and clinical science with the goal of creating 
new tissues and organs. This is a science based on fundamental principles that involve the identification of the corresponding cells, the development of the scaffold-carrier and the morphogenic signals needed to induce cell regeneration of the tissue or organ. Over the past few years medicine has begun to investigate the possibility of stem cell and tissue engineering application in order to repair and regenerate damaged tissues and organs. This type of therapy is becoming part of the medicine called regenerative medicine, which already occupies a significant place in clinical practice. The concept of tissue engineering is integrated into the research and application of regenerative dentistry, including periodontology, endodontics and maxillofacial surgery, all of which aim to treat damaged and lost oral tissue through the reconstruction and regeneration of periodonium, dentin-pulping complex and orofacial tissue. The three essential components required for tissue engineering include a population of multipotent progenitor cells, the presence of signal molecules/inductive morphogenic signals and a conductive extracellular scapular matrix. The extracellular scaffold matrix does not only act as a means of delivering cells to the site of regeneration, but also plays a major role in cell assembling, keeping space, determining morphological properties and using oxygen and nutrients. Factors of great importance in the selection of a suitable scaffold material include features such as porosity, tissue conductivity, biocompatibility and resorption rate. Perhaps the most critical component of tissue engineering is the choice of stem cell population. Progenitor cells are of particular interest in the healing of periodontal wounds and regeneration, since most likely the parent cells from which osteoblasts, cement fibers and fibroblasts are formed are responsible for the restoration of lost periodontal tissues (Figure 1) [2].

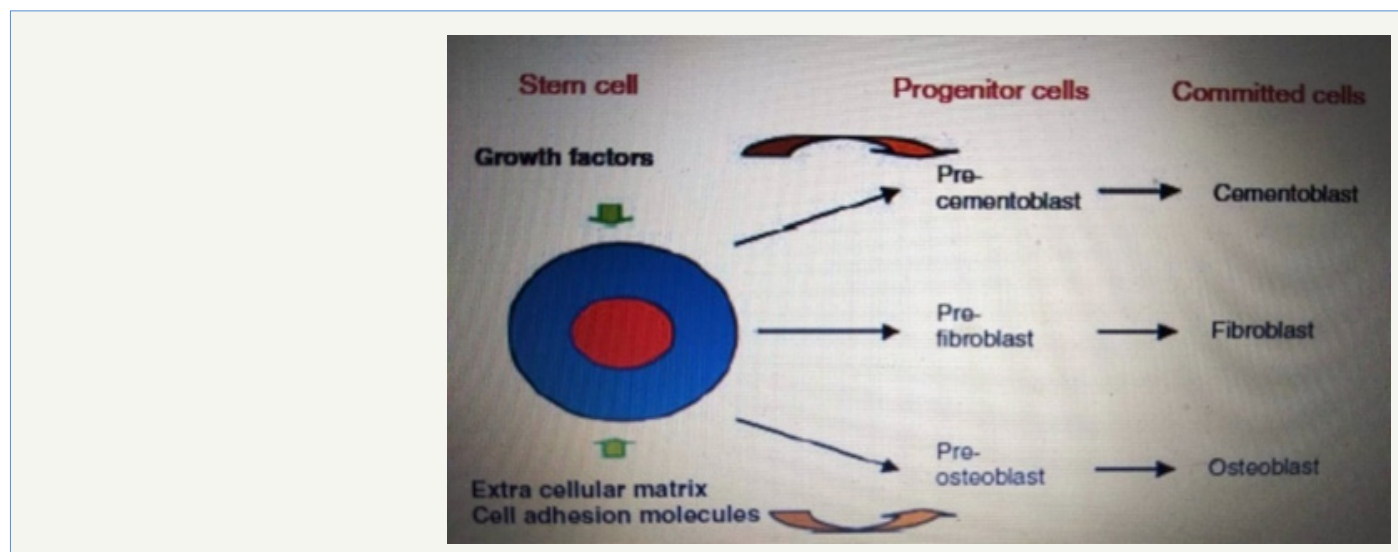

Figure 1: A schematic diagram of the proposed differentiation of adult mesenchymal stem cells and progenitor cells into periodontal cells.

\section{Stem cells}

Stem cells are undifferentiated cells with varying degrees of potential and plasticity, capable of self-regeneration and multipurpose differentiation. To date, stem cells have been isolated from three main sources: embryonic stem cells, somatic stem cells (adult stem cells, mesenchymal stem cells) and, more recently, through genetic manipulation induced pluripotent stem cells (iPSc). While the use of embryonic stem cells is limited by ethical issues, somatic stem cells constitute a more favorable cellular source used in tissue engineering. Postnatal stem cells are isolated from several tissues including brain, skin, hair follicles, skeletal muscles, bone marrow and dental tissue [2]. Five types of stem cells are isolated from the tooth: Dental pulp Stem Cells (DPSC) of permanent teeth; stem cells from exfoliated deciduous teeth (SHED); periodontal ligament stem cells (PDLSCs); apical papillary stem cells (SCAP) and progenitor cells of dental follicles (DFPC) [3].

Some stem cells have the ability to differentiate into all types of tissue, while others can only be differentiated into a limited number of tissues. According to differentiation capabilities we distinguish:

A. Totipotent - can be differentiated into all types of cells and tissues (fertilized eggs);
B. Pluripotent - can be differentiated into most cells and tissues (embryonic stem cells);

C. Multipotent - mesenchymal stem cells;

D. Oligopotent - myeloid stem cells;

E. Unipotent - skin.

In general, adult stem cells are more limited in their differentiation capabilities compared to embryonic stem cells known to form tumors when implanted in vivo. Stem cells of adults appear as the main candidate for use in regenerative therapies because their isolation and use does not require the same level of legal and ethical issues as embryonic stem cells. The further advantage of adult stem cells is the greater capacity for their use in autologous transplantation where adult stem cells can be derived from the patient and then used to treat this patient by reducing the likelihood of complications from immunological rejection [2].

Mesenchymal stem cells (MSCs): Mesenchymal stem cells first described Friedenstein as clonogenic, derived from bone marrow, plastic, adherent cells. Since discovery, these cells are called stromal bone marrow cells and stromal cell precursors, recently, mesenchymal stem cells. The Committee of Mesenchymal and Tissue Stem Cells of the International Society of Cellular Therapy proposed 
the minimum criteria for defining multipotent mesenchymal stem cells. Their scheme includes three criteria for the definition of mesenchymal stem cells: adhesion to the plastic substrate adherence; specific pattern of surface antigen expression; and multipotent potential of differentiation. In particular, mesenchymal stem cells must be plastic-adherent when cultured under standard cultivation conditions in standard cultures. At least 95\% of the population of mesenchymal stem cells must also express CD105, CD73 and CD90. Furthermore, $<2 \%$ of the population should display markers CD45, CD34, CD14 or CD11b, CD79a or CD19 and HLA Class II. Finally, the population of mesenchymal stem cells must be able to penetrate through three lines of mesenchymal differentiation for the formation of osteoblasts, adipocytes and chondroblasts under standard in vitro differentiation conditions. Only cells that show all these characteristics can be described as mesenchymal stem cells. However, it has to be emphasized that the immunophenotype described herein is also expressed in the culture of fibroblasts isolated from different tissues that have small or no multi-cellular potential of differentiation [1]. Mesenchymal stem cells are one of the most studied types of adult stem cells. They can be differentiated into different types of cells and tissues. Mesenchymal stem cells have a lower development potential and a shorter lifespan than pluripotent embryonic stem cells, which have the ability to proliferate unlimited in vitro and the potential to differentiate into all types of cells present in the body.

Thanks to their availability, high capacity for growth and multipotency, mesenchymal stem cells are very promising for tissue regeneration in clinical applications. However, there are several technical problems that still need to be overcome, including the identification and isolation of the appropriate types of precursors, the establishment of optimal growth and differentiation in vitro and effective delivery conditions for the successful transplantation of mesenchymal stem cells. Essential components for generating an effective therapeutic strategy based on the cells include a population of multipotent progenitor cells, the presence of signal molecules / inductive morphogenic signals and a conductive extracellular scapular matrix or an appropriate delivery system. Initial studies have shown that mesenchymal stem cells can be obtained from bone marrow, spleen and thymus. Subsequent studies identified mesenchymal stem cells from trabecular bone, periosteum, articular cartilage, synovium, synovial fluid, skeletal muscle, fatty tissue, tendon, blood, blood vessels, umbilical cord, placenta, fetal tissue and skin. Mesenchymal stem cells are also isolated from dental tissues, including periodontal ligament, tooth pulp, exfoliated deciduous teeth, apical papilla and tooth follicles [1].

Mesenchymal stem cells of the bone marrow: The bone marrow consists of three main cellular systems: hematopoietic, endothelial and stromal. These systems are derived from at least three different populations of stem cells present in the bone marrow: haematopoietic stem cells from which blood cells are formed; endothelial precursor cells known as angioblasts; and different stromal populations derived from stromal /stem cell bone marrow. In vivo, osteogenic bone marrow stem cell capacity was assessed primarily by transplantation of cultured bone marrow stem cells subcutaneously into the dorsal surface of the immunodeficiency mice in combination with an osteoconductive mixture of hydroxyapatite / tricalcium phosphate carriers. A histological examination of the transplant shows a wide array of blood vessels and fibrous tissue as a result of a newly developed bone formation. The cell material in newly formed bone tissue in vivo is confirmed by human origin, in situ hybridization using a human specific Alu-genetic sequence. In their pioneering study, Bruder et al. assessed the repair potential of bone marrow stem cells in bone tissue of large animals. Using bone marrow stem cells for reparation of femoral bone defect in dogs, the formation of newly created bone and healing at the defect site were demonstrated. Bone marrow stem cells have been intensively studied in terms of their periodontal regeneration capacity. Obviously, bone marrow stem cells have the capacity to improve periodontal regeneration through improved formation of well-formed mature alveolar bone and neovascularization. Moreover, the transplanted bone marrow stem cells contributed to the formation of a new cement and periodontal ligament. While it has been proven that bone marrow stem cells have the capacity to regenerate periodontal tissue, the difficulties associated with bone marrow stem cell isolation pose a challenge for the use of this cell population in periodontal regeneration in clinical practice [1].

Dental pulp stem cells: The regenerative capacity of the human dentin / pulp complex revealed to scientists that the dental pulp contains progenitor cells responsible for reparation of dentine. Gronthos first identified adult dental pulp stem (DPSC) cells in human dental pulp in 2000. and found that DPSC can regenerate the dentin-pulse complex, which consists of a mineralized matrix with coated tubes with odontoblasts and a connective tissue containing blood vessels in the arrangement a similar dentin-pulping complex found in normal human teeth [4]. The same group further confirmed that the DPSC possesses striking self-renewal and multilinear differentiation capabilities, finding that DPSC are able to form ectopic dentin and accompanying pulp tissue in vivo and to differentiate into adipocytes and neurons of a similar cell [4]. An in vivo study has shown that DPSC produces bone when implanted on subcutaneous sites in immunocompromised mice with HA / TCP powder as a carrier. In addition, the potential of DPSC for longterm storage was analyzed. It has been discovered that even after 2 years of storage, DPSC continued to be able to differentiate into preosteoblasts and produce bone tissue. In addition, DPSC continued to express certain surface antigens, confirming cell integrity. A few in vitro and in vivo studies have been well demonstrated by the osteogenic differential capacity of the DPSC. A strong expression of alkaline phosphatase and the formation of mineralized nodules can also be induced during the osteogenic differentiation of isolated STRO-1* DPSCs (Figure 2) [5]. The scientists worked to find an effective scaffold that could be filled with DPSC and an appropriate microenvironment to promote the differentiation of DPSC. In a recent study DPSCs were seeded on various 3-dimensional (3-D) scaffolds (spongy collagen, porous ceramics and fibrous titanium 
mesh) and implanted in mice for 6 or 12 weeks, the formed tissue was not a dentin-pulse complex but it resembled connective tissue. These studies show the potential of DPSC in dental tissue engineering [6]. Although the tooth pulp is subjected to calcification by various stimuli, the precise cell population that has the ability to form hard tissue in the pulp remains unknown. It is believed that these cells in the tooth pulp are localized or in a perivascular niche or in a layer rich in sub-cellular cells. The tooth pulp contains undifferentiated cells expressing markers of stem and progenitor cells such as STRO-1, ABCG2, CD90, alpha-smooth muscle actin and Bmi1. In addition, STRO-1 and ABCG2-positive cells were recorded in a perivascular niche under normal physiological conditions; On the other hand, most cells in the sub-odontoblast layer exhibit CD90 [7]. The potential of stem cells to repair bone defects in humans was tested in a clinical study. The aim of this study was to treat alveolar bone defects routinely associated with the extraction of an impacted, non-erupted or partially erupted wisdom tooth. At the time of extraction of the autologous stem cell of the tooth pulp previously obtained from the above molars of these patients in combination with the collagen carrier, they were implanted into the space of the tooth extracted to prevent the postoperative loss of the alveolar bone. The results of this study indicate autologous bone regeneration since this procedure led to optimal bone regeneration with periodontal tissue restoration in the desired area. Together, these results indicate the potential role of pulp stem cells in periodontal regeneration in addition to bone and dentine regeneration; However, in this case much more research is required [1].
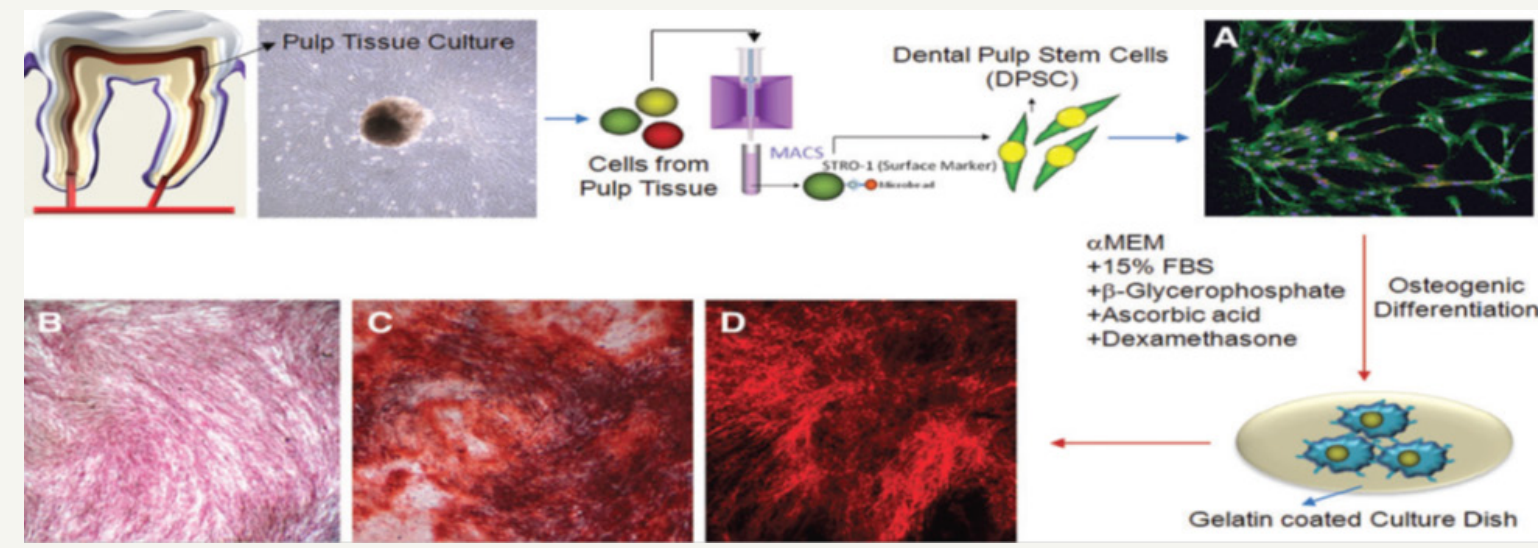

Figure 2: In vitro osteogenic differentiation of DPSC. Stem cells are isolated from the tissue of the tooth pulp by purifying the STRO-1-positive cell population with the MACS system, and are allowed to differentiate into osteogenic lines to form mineralized nodes under the proper conditions of osteogenic culture. (A) Immunocytochemical image of isolated DPSC: Stro-1 (green), CD44 (red), and nuclear staining (DAPI, blue). (B) Phenotypic expression of alkaline phosphatase after 21 days of osteogenic culture. (C) Alizarin red-S-colored image of mineralized nodules after 21 days of osteogenic culture under a light microscope. (D) Alizarin red-S-colored image of mineralized nodules after 21 days of osteogenic culture under a fluorescence microscope.

Stem cells of the periodontal ligament: Periodontal ligament (PDL) is a specialized connective tissue that connects the surface of the tooth root with the alveolar bone. PDL has a heterogeneous population of cells that can be differentiated into cement or bone cells. The periodontal ligament stem cells (PDLSCs) are capable of self-regenerating and expressing the markers of mesenchymal stem cells CD146 / MUC18, CD105, CD166 and STRO-1. PDLSC cells also express the tendon-specific markers. These data indicate that PDLSC is a unique population of post-natal stem cells different from dental pulp stem cells or mesenchymal stem cells derived from the bone marrow. Similar to stem cells derived from pulp, PDLSC are multipotent cells with high potential for osteogenic, adipogenic and chondrogenic differentiation. When transplanted with HA / TCP into immunodeficiency mice the PDLSC produce a cement-like / PDL-like structure and contribute to the repair of periodontal tissue. Although there was no evidence that PDLSCs form pulp of similar structure, when they were implanted with SCAP to HA / TCP and gel-foam networks in mini-pig models, bio-roots with functional PDL were obtained $[3,8]$. Under the same conditions it was found that stem cells of the periodontal ligament produced a higher amount of fibroblast than those described in bone marrow stem cells and tooth pulp. The level of sclerosis expression in stem cells of periodontal ligament is higher than in bone marrow stem cells and dental pulp stem cells, suggesting that periodontal ligament stem cells exhibit unique properties compared to other mesenchymal stem cells [1]. The transplantation of periodontal ligament stem cells multiplied ex-vivo in immunocompromised mice confirmed the ability of these cells to form functional, cement-like similar cells and cement / periodontal ligament-like tissues, including Sharpe's fibers, in vivo. Significant studies have been carried out to assess the regenerative capacity of stem cells periodontal ligaments in the management of dental defects in various animal models and in a single clinical study. In this clinical study, the potential of progenitor cells of the periodontal ligament was studied in bone defects in three patients. A retrospective comparison of the progenitor of the periodontal ligament stem cells has shown that these cells have smaller osteogenic and adipogenic differentiation capacity in vitro. However, these cells have a slightly higher migratory potential in vitro. Furthermore, autologous progenitors of the periodontal ligament mixed with bone grafting material and implanted in deep periodontal pockets of patients monitored during $3,6,12,26$, 32,42 and 72 months showed that two out of three patients had 
noticeable regeneration tissue. The third patient had reduced tooth mobility and depth of pocket, reduced gingival recession and stable improvement of attachment. This study concluded that autologous periodontal ligament progenitors in cell-based surgical treatments for periodontitis can be effective in regenerative dentistry. In another study was observed the migratory capacity of stem cells of periodontal ligament (Figure 3). The cell migration capacity to the injured area was observed after 12 hours from the beginning of the lesion; Cells spread between $10-20 \%$ of the critical area. After 24 hours, the number of cells was increased and then they covered 40 $60 \%$ of the surface of the lesion. After 48 hours the cells were semi- confluent; after 72 hours the cells completely covered the area, which can be seen in Figure 3 [9]. In general, it has been shown that stem cells of periodontal ligament have the potential to form bone, cement and periodontal ligament of a similar structure and to improve general periodontal regeneration. So far, periodontal ligament stem cells appear to have a higher capacity to generate dental structures compared to other mesenchymal stem cells like cells, making them extremely acceptable for use in periodontal regeneration [1]. again in 40-60\%. D) and E) after 48 hours, it can be noted that the cells covered $80-90 \%$. F) 72 hours after the lesion, the cells again reached confluence.
A)

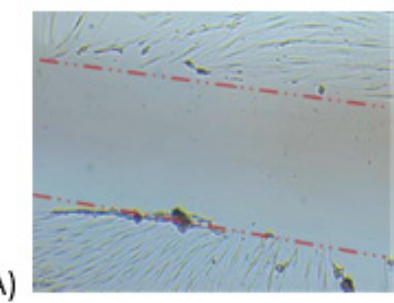

D)

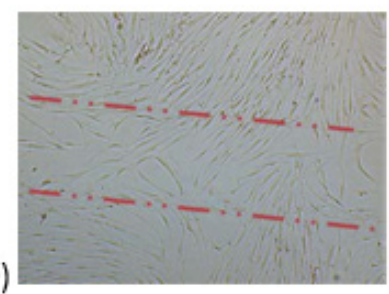

B)

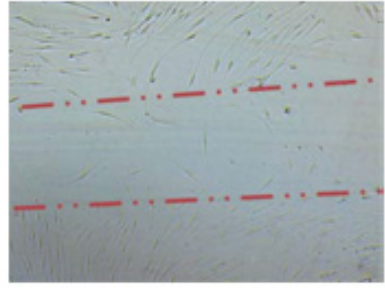

E)

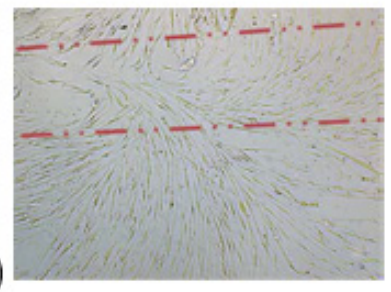

C)

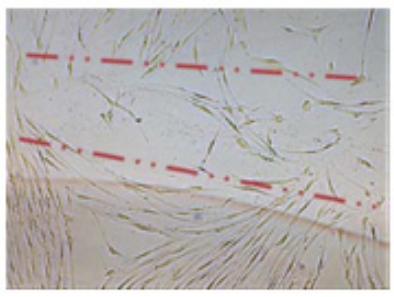

F)

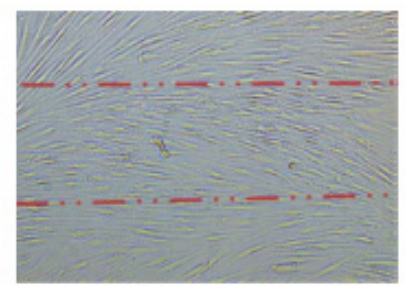

Figure 3: A) A photograph taken immediately after the establishment of a lesion in culture. B) 12 hours after the lesion, migration of the cell group, again colonize 10 to $20 \%$ of the surface of the lesion. C) 24 hours later cell group migration was observed to regenerate the damaged area again in $40-60 \%$. D) and E) after 48 hours, it can be noted that the cells covered $80-90 \%$. F) 72 hours after the lesion, the cells again reached confluence.

Stem cells from human exfoliated deciduous teeth: Stem Cell Detection in Deciduous Teeth [10], throws a new light on the intriguing possibility of using stem cells tissue in tissue engineering. The obvious advantages of SHED (stem cells from human deciduous teeth) are: a higher rate of proliferation compared to stem cells of permanent teeth, easily proliferate in vitro, high plasticity until they differentiate into neurons, adipocytes, osteoblasts and odontoblaste, easy accessibility in young patients [10], especially suitable for young patients with mixed dentition. Miura has shown that SHEDs cannot be directly differentiated into osteoblasts, but have induced the creation of new bones by forming a form for the recruitment of osteogenic host cells [10]. SHEDs are characteristic of osteoinduction and high plasticity. Cordeiro sowed SHED in PLLA (porous poly-L-lactic acid) prepared with scapular and transplanted into subcutaneous tissue of immune-deficient mice. They noted that SHEDs were differentiated into odontoblast cells and showed morphological characteristics similar to those in the odontoblast cells. It was also confirmed that transplanted SHEDs were able to differentiate into blood vessels that anastomized with host vasculature [11]. These studies have shown that SHED can be the ideal source of stem cells to repair damaged tooth structures and cause bone regeneration [11]. Isolation and characterization of in vitro stem cells from human decidous teeth is derived from coronary pulp of decidous teeth. in vitro, stem cells from human decidous teeth are multipotent stem cells that are able to differentiate into a series of mesenchymal and non-mesenchymal cell types, including nerve cells, adipocytes, chondrocytes and odontoblasts. In vivo, stem cells from human decidous teeth have the ability to cause bone formation, generate dentine, generate dental pulp tissue, express neuronal markers and survive in the mouse's brain [1]. There are few publications of stem cells implantation from human deciduous teeth to periodontal defects; However, they were used to regenerate the tissue of the tooth pulp when implanted within human teeth placed in immunodeficiency mice. While stem cells from human decidous teeth were not implanted into periodontal defects, the equivalent stem cells derived from the decidous teeth of miniature pigs were. Zheng et al. implanted stem cells derived from the decidous teeth of miniature pigs in the bone with a critical defect size created in the parasymphysis region of the mandible. Their study showed that the implanted stem cells were directly differentiated into the new bone, resulting in a significantly higher formation of the new bone at the defect site. In addition, Yamada et al. performed allogeneous transplantation of stem cells in dogs in combination with platelets trombocites in bone defects of the 
mandible. Implanted cells generate a well-formed mature bone that was neovascularized at defect sites. Together, these studies provide evidence that human deciduous stem cells can serve as an alternative source of stem cells for stem cell-based treatment for periodontal regeneration [1].

Stem cells of apical papilla: Stem cells are also found in the top of the root of the teeth. These cells are called apical papilla stem cells (SCAP) and have been shown to differentiate and demonstrate higher in-vitro proliferation rates than DPSC. There is an apical zone rich in cells that lies between apical papilla and pulp. It is important to emphasize that parent / progenitor cells are also found in dental pulp and apical papilla but have slightly different characteristics. The higher proliferative potential of SCAP makes this population of cells more suitable for cell-based regeneration and predominantly for the formation of roots. They are able to form odontoblasts like cells and produce dentin in vivo and are likely to be the source of primary odontoblast cells for the formation of root dentin. The discovery of SCAP can also explain the clinical phenomenon presented in several recent reports of clinical cases that show that apexogenesis can continue to exist in infected, undermature, permanent teeth with apical periodontitis or abscess. It is likely that SCAP living in apical papilla survives infection due to their proximity to periapical tissues. Perhaps, after endodontic disinfection, these cells can lead to the formation of primary odontoblasts in order to complete the formation of the root. [3]. The apical papilla stem cells are a unique population of multipotent stem cells because they express a high level of expression of the two genes involved in the mediation of cell proliferation: survivin and telomerase [1]. Stem cells from apical papilla transplanted into immunocompromised mice formed a dentin-like layer of tissue on the surface of hydroxyapatite / tricalcium phosphate, which was used as a cell carrier. The authors of this paper suggest that dentin was produced in vivo formed from odontoblasts like cells that were differentiated from cells similar to mesenchymal stem cells present among apical papilla stem cells. The apical papilla stem cells were transplanted with stem cells of the periodontal ligament into the dental alveolus of miniature pigs in order to regenerate the root and form a tissue like the periodontal ligament. The results of this work have shown that together these stem cells can generate a root / periodoncy complex capable of supporting the porcelain crown and restoring the normal tooth function. More work is needed on the use of apical papilla stem cells before conclusions are drawn about their usefulness in the treatment for periodontal regeneration [1].

Stem cells of dental follicles: Dental follicle is a loose connective tissue of ectodermal origin that surrounds a tooth-germ in development, pre-eruption. Dental follicles were considered to contain progenitors for cementoblasts, periodontal ligament and osteoblast cells. Human dental follicle stem cells are most often isolated during the extraction of the impacted third molars. Stem cells isolated from human dental follicles are characterized by their fast binding to culture and the expression of markers typical of stem cells-nestin and notch-1. The stem cells of the dental follicles can be leaded in the direction of adipogenic, osteogenic and neurogenic differentiation in vitro. Dental follicle stem cells can also form compact calcified nodules in vitro, suggesting that these cells can be differentiated into cement. The stem cells follicles in vivo have the ability to differentiate into odontoblasts and contribute to the formation of dentine in the presence of a dentin matrix scaffold. Dental follicles stem cells also have the capacity to create a periodontal ligament in vivo. Guo et al. used stem cell follicles to assess the ability of such cells to contribute to the formation of the root of the teeth. They implanted the stem cell follicles in three different micro-circuits in the rat: an uninfected pigmental pocket, a highly mineralized skull, and an inductive alveolar foss. Stem cells of dental follicles are implanted at these locations in combination with a dentin matrix-treated scaffold. Stem cells of dental follicles contributed to the regeneration of dentine within the pockets and contributed to the formation of a mineralized matrix in skull defects. Interestingly, stem cells follicles implanted in alveolar fossa contributed to the formation of tissues like the pulpo-dentine complex root and periodontal ligament associated with a cementlike layer covering the alveolar bone. These results show that the micro-environment in which the stem cells implant affects the capacity of these cells in the formation of various tissues. More interesting, these results also demonstrate the potential that dental follicle stem cells have in the tooth root regeneration [1].

\section{Induced Pluripotent Stem Cells (iPSCs)}

In 2006 it was discovered that stem cells can be generated from adult somatic cells through the cellular reprogramming process [12]. Generated stem cells with this new technology called induced pluripotent stem cells (iPSCs). Since their discovery, there is a significant interest in iPSC because this technology makes it easier to generate adult human pluripotent stem cells without the need for human embryos, which circumvent a series of ethical and legal issues that hinder the research of embryonic stem cells to date [12]. IPSC appears to have a number of advantages over other dental stem cells, such as those derived from the periodontal ligament (PDL) and tooth pulp. In particular, iPSCs can be generated from easily accessible sources - tissues, including oral mucosa and gingival tissue. More importantly, iPSCs are very proliferative, which allows the acquisition of a large number of stem cells that would be needed for use in regenerative therapy. However, before using iPSCs in the main procedures in dentistry, some important facts need to be considered. The main disadvantages of iPSC include their genomic instability and their tendency to form tumors in vivo. While there are gaps associated with iPSCs, the potential that iPSCs have shown in the treatment of multiple disorders require further research in order to minimize related therapeutic risks. The use of virally integrated vectors in generating iPSCs is a factor that contributes to genomic instability and tumorigenic potential of iPSCs. However, tumorigenic potential of iPSc can be reduced by differentiating iPSc into linear specific progenitor cells or mature cell populations prior to their use in regenerative therapies. An analysis of the tumorigenicity of mesenchymal stem cells derived from iPSc (iPSC-MSCs) in NOD / SCID mice failed to identify the formation of any tumors [13]. 
Considering the enormous potential of iPSC in dental research and the potential benefits of using dental iPSC, many researchers have reprogrammed cell populations derived from dental tissue into pluripotent stem cells. Figure 4. summarizes dental tissues that have been successfully reprogrammed in iPSCs. IPSCs were successfully generated from tooth pulp cells derived from exfoliated deciduous teeth, stem cells from apical papilla and dental pulp stem cells; cells from extracted human wisdom teeth; immature dental pulp stem cells; dental pulp cells of naturally lost teeth; oral mucosa-fibroblasts; gingival tissue and PDL stem cells. To date, several studies have investigated the potential utility of iPSC in various dental applications, including periodontal regeneration and tooth development and regeneration. Duan et al. [14] have investigated the potential of human iPSCs for the regeneration of periodontal tissues during implantation in a surgically created periodontal defect. Addition of iPSc results in a significantly higher alveolar bone, cement and PDL regeneration formation in mice that received iPSC in combination with silk scapular and eye matrix derivatives (EMDs) compared to control mice that received silk skafold and EMD or only silk scapular. This study identified the ability of iPSc to contribute to periodontal regeneration without any adverse reactions, although it remains to determine whether iPSc alone without a skafold or EMD can achieve the same results [13]. To reduce or completely eliminate the tumorigenic potential of iPSc prior to clinical use, they approach their differentiation. The most commonly implemented two differentiation approaches that are particularly relevant for dentistry - differentiation of iPSC into MSC and differentiation of iPSC into osteoprogenitors cells. Figure 5. shows the potential of using differentiated iPSCs in dentistry.

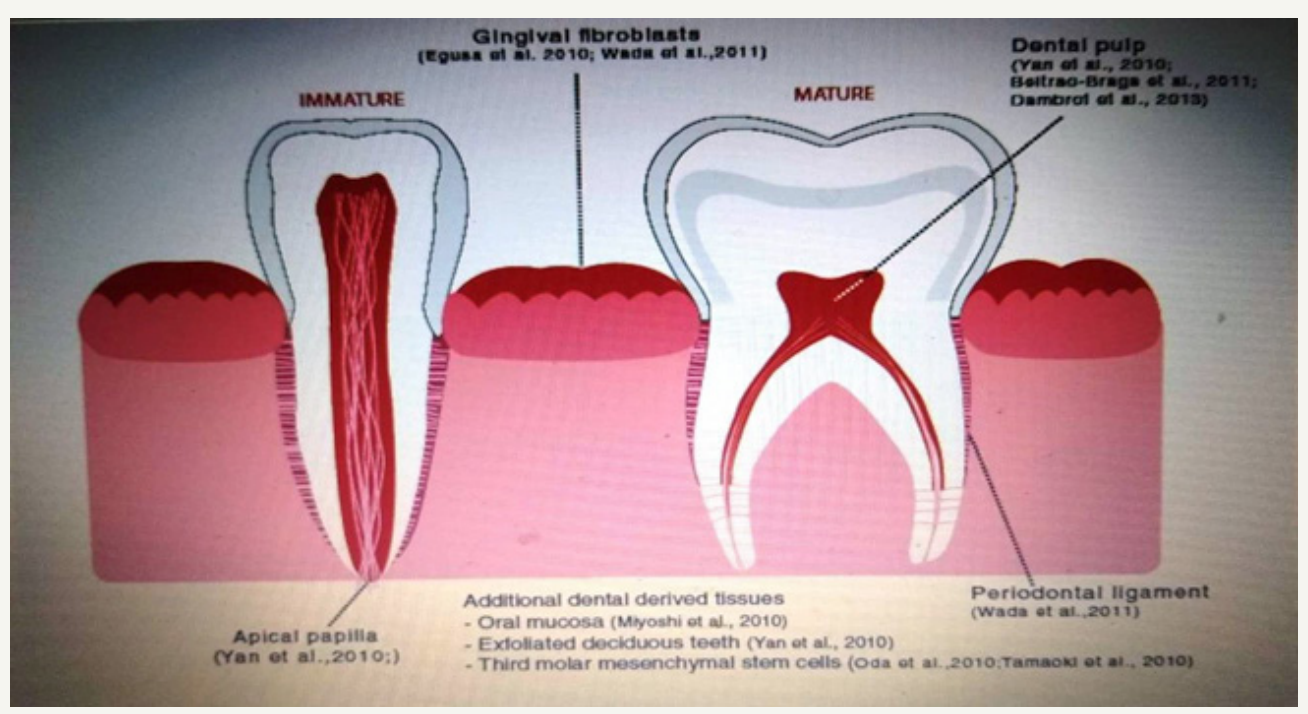

Figure 4: Oral tissues from which the induced pluripotent stem cells (iPSc) can be successfully isolated.

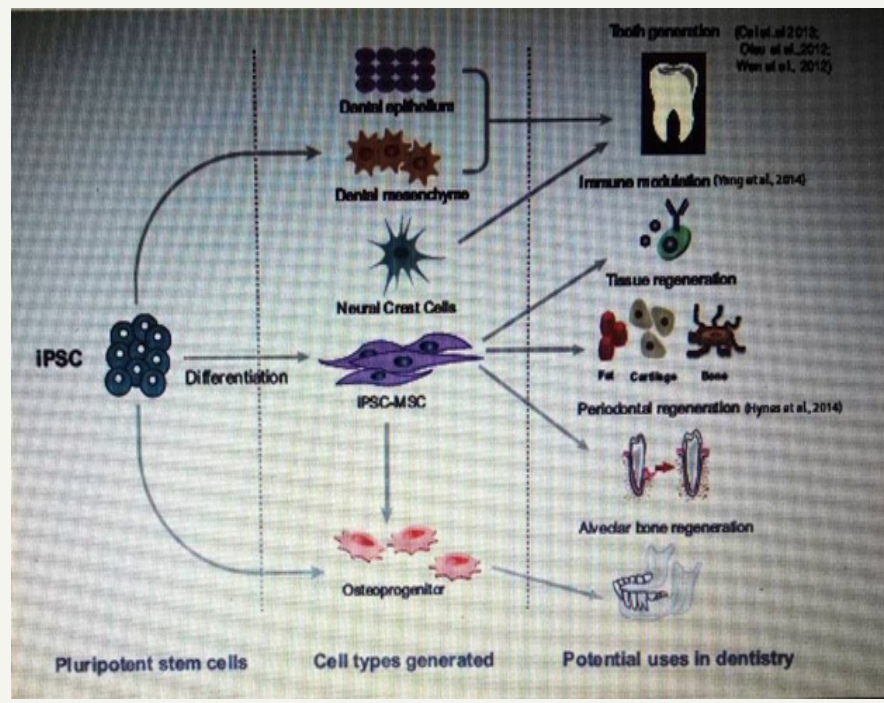

Figure 5: Potential use of differentiated induced pluripotent stem cells (iPSCs) in dentistry. 
This picture summarizes the potential of clinical use of iPSCs in dentistry. Given the tumor potential of iPSc, it is unlikely that undifferentiated iPSCs will be safe for clinical use. However, it has been shown that differentiation of iPSc into multiple cellular populations of restricted lines is much safer option. To date, 3 main proposals for the use of iPSC and their derivatives in dentistry have been proposed and / or explored. First, iPSC showed the potential for use in tooth formation. Numerous cell populations are involved in tooth formation - namely, dental epithelium, dental mesenchymes and cells such as neuronal ridge and iPSC can be used to create these cell populations which, when combined, can facilitate the formation of teeth. Another approach to the use of iPSC includes their differentiation into mesenchymal stem cells (iPSC-MSCs), which can then be used in dental applications directly or in the production of osteoprogenitor cells. To date, iPSCs-MSC have demonstrated their immune-modulation potential and tissue and periodontal regeneration [13]. Finally, iPSCs and iPSCsMSCs have been successfully differentiated into osteoprogenitor cells, and while these cells have yet to be evaluated in the dental environment, it is probably only a question of their use time [13] A recent study by Hines et al. [13] assessed the ability of iPSC-MSC to contribute to regeneration of the periodontal tissue. To achieve this, the authors used bone defects in rats, in which a surgical defect was created by removing the bone covering both the first and the second molar. IPSC-MSC were delivered via a fibrinogen and a thrombin clot and implanted in a phenestration defect. Two weeks after implantation, the level of tissue regeneration in the phenestration defect was assessed through histomorphometric analysis. The authors identified a significant increase in the amount of newly formed mineralized tissues and PDL-like tissues present within the defect in those animals receiving iPSC-MSC compared to the control defect. In addition, the authors could demonstrate that the implanted iPSC-MSCs were grown, differentiated and stimulated regeneration of the periodontal tissue. These results have shown that iPSC-MSCs are a promising source of readily available stem cells for use in periodontal regeneration [13].

Also, transplantation of the iPS cells showed higher levels of OC, Osk and Runk2 gene expression, both 12 and 24 days after surgery. At the 24th day after the surgical procedure in the iPS cell group, the histological analysis showed a much larger newly formed formation of alveolar bone and cement with regenerated periodontal link between them [14]. Another study evaluating the usefulness of iPSC-MSC in the treatment of periodontitis has used combined ligatures and infections as a model of periodontitis The results show that iPSC-MSC is able to reduce inflammation in experimental periodontitis and may serve as an alternative source of stem cells for the treatment of periodontitis. Together, these two studies show significant potential for iPSC-MSC in the treatment of periodontitis, that iPSC-MSC have the ability to facilitate dental tissue regeneration [13] and inhibit the chronic inflammatory response that causes tissue damage [13].

\section{Conclusion}

The use of stem cells in periodontal regeneration is very promising. However, as with all new technologies, questions often arise faster than answers. The time has elapsed to move from animal studies to clinical studies on humans. Many studies conducted on animals give indisputable evidence that mesenchymal stem cells can be successfully used in periodontal regeneration. There are several major tasks to be addressed before developing effective cell therapy for regenerative dentistry. First of all, a better understanding of: (a) self-reproducibility mechanisms to regulate the growth of adult stem cells in vitro to generate the required number of cells for different applications; (b) stem cell regulation during differentiation and maturation in specific cell types as well as during wound healing; (c) interactions between stem cells and the immune system, in particular with regard to the use of populations of allogeneic cells; and (d) the mechanisms necessary for the control and prevention of ex vivo-expanded mesenchymal stem cells from unwanted transformation. Issues such as appropriate delivery devices, immunogenicity, autologous cells versus allogeneic cells, tissues are most appropriate as donors, control of the whole process and cost-effectiveness are also those that need to be considered. In addition, before we are completely sure, the next critical phase is the systematic validation of specific mesenchymal stem cells as a reliable source for cytotherapeutic use. Finally, the establishment of key rules for the preparation and taking of stem cells that contain stringent good manufacturing practice protocols will be an absolute necessity.

\section{References}

1. Hynes K, Menicanin D, Gronthos S, Bartold PM (2012) Clinical utility of stem cells for periodontal regeneration. Periodontology 2000 59(1): 203-227.

2. Han J, Menicanin D, Gronthos S, Bartold PM (2014) Stem cells, tissue engineering and periodontal regeneration. Aust Dent J 59(Suppl 1): 117-130.

3. Estrela C, Alencar AHG, Kitten GT, Vencio EF, Gava E (2011) Mesenchymal stem cells in the dental tissues: Perspectives for tissue regeneration. Braz Dent J 22(2): 91-98.

4. Gronthos S, Mankani M, Brahim J, Gehron Robey P, Shi S (2000) Postnatal human dental pulp stem cells (DPSCs) in vitro and in vivo. Proc Natl Acad Sci U S A. 97(25): 13625-13630.

5. Li Peng, Ling Ye, Zhou XD (2009) Mesenchymal stem cells and tooth engineering. Int J Oral Sci 1(1): 6-12.

6. Kim BC, Bae H, Kwon IK, Lee EJ, Park JH, et al. (2012) Osteoblastic/ Cementoblastic and neural differentiation of dental stem cells and their applications to tissue engineering and regenerative medicine. Tissue Eng Part B Rev 18(3): 235-244.

7. Hosoya A, Nakamura $\mathrm{H}$ (2015) Ability of stem and progenitor cells in the dental pulp to form hard tissue. Japanese Dental Science Review 51(3): 75-83.

8. Demarco FF, Conde MCM, Cavalcanti B, Casagrande L, Sakai V, et al. (2011) Dental pulp tissue engineering. Braz Dent J 22(1): 3-13.

9. Iriarte CGT, Ramírez OR, García AM, Terán SLV, Clavel JFG (2017) Isolation of periodontal ligament stem cells from extracted premolars. Simplified method. Revista Odontológica Mexicana 21(1): e12-e20.

10. Miura M, Gronthos S, Zhao M, Lu B, Fisher LW, et al. (2003) SHED: Stem cells from human exfoliated deciduous teeth. Proc Natl Acad Sci U S A 100(10): 5807-5812.

11. Cordeiro MM, Dong Z, Kaneko T, Zhang Z, Miyazawa M, et al. (2008) Dental pulp tissue engineering with stem cells from exfoliated deciduous teeth. J Endod 34(8):962-969. 
12. Takahashi K, Yamanaka S (2006) Induction of pluripotent stem cells from mouse embryonic and adult fibroblast cultures by defined factors. Cell 126(4): 663-676.

13. Hynes K, Menichanin D, Bright R, Ivanovski S, Hutmacher DW, et al. (2015) Induced pluripotent stem cells: A new frontier for stem cells in dentistry. J Dent Res 94(11): 1508-1515.
14. Duan X, Tu Q, Zhang J, Ye J, Sommer C, et al. (2011) Application of induced pluripotent stem (iPS) cells in periodontal tissue regeneration. J Cell Physiol 226(1): 150-157. (c) (i) Creative Commons Attribution 4.0

For possible submissions Click Here Submit Article

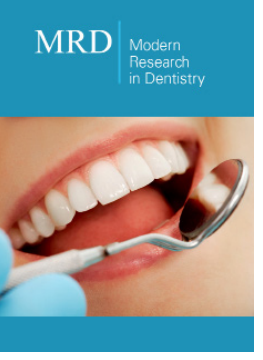

\section{Modern Research in Dentistry}

Benefits of Publishing with us

- High-level peer review and editorial services

- Freely accessible online immediately upon publication

- Authors retain the copyright to their work

- Licensing it under a Creative Commons license

- Visibility through different online platforms 